Morais, MHL, Souza, WM \& Ribeiro, AJA. (2020). Use of red ceramic waste as fine aggregate in concretes. Research, Society and Development, 9(7): 1-17, e357974145.

\title{
Utilização dos resíduos de cerâmica vermelha como agregado miúdo em concretos
}

Use of red ceramic waste as fine aggregate in concretes

\section{Uso de residuos de cerámica roja como agregado fino en concreto}

Recebido: 01/05/2020 | Revisado: 05/05/2020 | Aceito: 08/05/2020 | Publicado: 17/05/2020

\section{Maria Helany Lima de Morais}

ORCID: https://orcid.org/0000-0002-2318-9040

Instituto Federal de Educação, Ciência e Tecnologia do Ceará, Brasil

E-mail: mariahelanyl@gmail.com

Wana Maria de Souza

ORCID: https://orcid.org/0000-0002-1269-6661

Instituto Federal de Educação, Ciência e Tecnologia do Ceará, Brasil

E-mail: wanamaria19@gmail.com

Antonio Júnior Alves Ribeiro

ORCID: https://orcid.org/0000-0002-6607-6409

Instituto Federal de Educação, Ciência e Tecnologia do Ceará, Brasil

E-mail: junior.ribeiro@ifce.edu.br

\section{Resumo}

O crescimento do setor da construção civil tem contribuído com um aumento significativo na geração de Resíduos Sólidos Urbanos (RSU). Tais resíduos acarretam diversos impactos ambientais em decorrência da sua disposição ambientalmente inadequada. Neste contexto, a incorporação dos Resíduos de Construção Civil (RCC) na área da engenharia vem se apresentando como uma alternativa técnica viável e a inovadora dos materiais de construção, sendo considerada vantajosa do ponto de vista técnico, econômico e ambiental. Diante do exposto, este estudo visa analisar a influência da substituição do agregado miúdo (areia natural) pelo Resíduo de Cerâmica Vermelha (RCV), através da Resistência à Compressão Simples (RCS) e do consumo de cimento Portland no concreto, de modo a reduzir os custos, bem como os impactos associados ao uso do concreto, tendo em vista o seu largo consumo. Para tanto, realizou-se três dosagens distintas pelo método do Instituto de Pesquisas Tecnológicas (IPT/EPUSP). Foram moldados dois corpos de prova cilíndricos para cada dosagem, totalizando dezoito corpos de prova. Logo, foram produzidos concretos com 
substituição parcial e total em termos de massa do agregado miúdo pelo resíduo supracitado, sendo estes percentuais de $0 \%, 50 \%$ e $100 \%$. Este procedimento foi feito para cada traço $(1: 3,5 ; 1: 5,0$ e 1:6,5). Os resultados mostraram que, em comparação ao traço de referência ( $0 \%$ de substituição), ocorreu o aumento da RCS aos 28 dias, bem como a diminuição do consumo de cimento Portland. Desta forma, infere-se que o RCV se mostra como um material alternativo potencialmente viável de ser utilizado na fabricação de concretos.

Palavras-Chave: Agregados de RCV; Concreto reciclado; Resistência mecânica; Consumo de cimento Portland.

\begin{abstract}
The growth of construction has contributed to a significant increase in the generation of urban waste. The inadequate disposal of this waste causes several environmental impacts. In this context, the use of urban waste as building materials is a viable and innovative technical alternative, being considered advantageous from a technical, economic and environmental point of view. This work aims to analyze the influence of the use of red ceramic waste (RCW) as a fine aggregate in the compressive strength (CS) and quantity Portland cement in concretes. For this, three different dosages were performed by the method of IPT / EPUSP, were produced with substitution of the fine aggregate by the red ceramic waste in the index $0 \%, 50 \%$ and $100 \%$. This procedure was done for each of the three mixtures of the method. The results showed that, in comparison to the reference mixture, there was an increase in CS at 28 days and a decrease in Portland cement consumption. Thus, the red ceramic waste analyzed, is shown as an alternative material potentially viable to be used in concretes.
\end{abstract}

Keywords: Portland Cement Consumption; Compressive Strength; Waste; Recycled concrete.

\title{
Resumen
}

El crecimiento de la construcción ha contribuido a un aumento significativo en la generación de residuos urbanos. La eliminación inadecuada de estos desechos causa varios impactos ambientales. En este contexto, el uso de residuos urbanos como materiales de construcción es una alternativa técnica viable e innovadora, que se considera ventajosa desde un punto de vista técnico, económico y medioambiental. El objetivo de este trabajo es analizar la influencia del uso del residuo de cerámica roja (RCR) como agregado fino, en términos de resistencia a la compresión simple (RCS) y en el consumo de cemento Portland, en la fabricación de concreto. Para este propósito, se llevaron a cabo tres dosis diferentes utilizando 
el método IPT / EPUSP, en el que se produjeron hormigones con reemplazo parcial y total en términos de masa del agregado fino por el residuo mencionado anteriormente, siendo estos porcentajes $0 \%, 50 \%$ y $100 \%$. Este procedimiento se realizó para cada línea $(1: 3.5 ; 1: 5.0$ y 1: 6.5) del método utilizado. Los resultados mostraron que, en comparación con el rasgo de referencia (reemplazo del 0\%), hubo un aumento en RCS a los 28 días y una disminución en el consumo de cemento Portland. Por lo tanto, parece que el RCJ se muestra como un material alternativo potencialmente viable para ser utilizado en la fabricación de concreto.

Palabras clave: Agregados RCJ; Concreto reciclado; Resistencia mecánica; Consumo de cemento Portland.

\section{Introdução}

O crescimento do setor da construção civil tem proporcionado um aumento significativo na geração de Resíduos Sólidos Urbanos (RSU), uma vez que estes representam em torno de $60 \%$ da composição dos RSU, dessa forma, acarretam diversos impactos ambientais em decorrência da sua disposição ambientalmente inadequada (Rosa, 2011; Souza, Xavier, Ribeiro \& Santos, 2019).

Inserido no setor da construção civil, destacam-se os materiais cerâmicos, que de acordo com Macedo, Menezes, Neves \& Ferreira (2008), a indústria deste material contribui com aproximadamente $1 \%$ no PIB do Brasil, ainda que, cerca de $40 \%$ desta contribuição é oriunda da produção de cerâmica vermelha, esta por sua vez, consome cerca de 70 milhões de toneladas de matéria-prima por ano.

Conforme Souza, Lima, Xavier \& Ribeiro, (2018), no que concerne ao índice de perdas nas indústrias de cerâmica vermelha, este oscila de $5 \%$ a $20 \%$, a depender do tipo de indústria. No Brasil, estima-se que o valor médio de perdas seja de 10\%, em decorrência da diversidade das cerâmicas. Já no estado do Ceará, esse percentual sobe para 20\%, um valor equivalente a, aproximadamente, $400 \mathrm{~kg}$ de Resíduo de Cerâmica Vermelha (RCV) por milheiro de peças fabricadas.

Segundo o Banco do Nordeste do Brasil (2010), o estado do Ceará produz 352.000 milheiros de peças cerâmicas mensalmente e a região do Cariri produz em torno de 70.506 milheiros por mês ( $20 \%$ da produção do estado). Considerando $20 \%$ de perda, o Cariri gera por mês 14.101 milheiros peças de RCV, considerando o peso médio de duas toneladas por milheiro, a região gera 28.202 toneladas de RCV por mês. 
A incorporação dos Resíduos de Construção Civil (RCC) na engenharia vem se apresentando como uma alternativa técnica viável e inovadora dos materiais de construção, sendo considerada vantajosa do ponto de vista técnico, econômico e ambiental, conforme descrevem os trabalhos de Melo (2005), Betat, Pereira \& Verney (2006), Cabral, Schalch , Molin \& Ribeiro (2009); Lima, Sales, Almeida, Moretti \& Portela, (2011), Silva (2014), Cabalar, Hanssan \& Abdulnafaa (2016), Possa \& Antunes (2016); Silva \& Carvalho (2017), Couto, Cezario, Morales \& Toralles (2018), Passos \& Moreno Júnior (2018), Souza et al. (2019).

A utilização de materiais alternativos voltados à produção de argamassas ou concretos que visam aprimorar o desempenho mecânico, ou ainda térmico, bem como a durabilidade desses materiais tem aumentado de forma significativa nos últimos anos. Tendo em vista a atenuação dos impactos ambientais correlatos à destinação final desses resíduos, bem como da extração de matéria prima (García, Junior, Quarcioni, \& Chotoli, 2015; Palhares, Pereira, Cabral \& Nobrega, 2016).

Em relação à utilização de resíduos mais finos (fíller) em substituições totais ou parciais em concretos e argamassas, ou ainda como materiais pozolânicos na produção de cimento Portland, pode-se citar os trabalhos de Leite (2001); Gonçalves (2007) Costa \& Favini (2008), Betat et al. (2009), Oliveira, Gomes \& Santos (2012), García (2014); Pereira, Silva, Queiroz, Moraes, Melges, Tashima, \& Akasaki (2015).

Pacheco-Torgal \& Jalali (2010) avaliaram a viabilidade do uso de resíduos de cerâmica no concreto. Os resultados apresentados conferiram que a incorporação dos agregados cerâmicos proporcionou um melhor desempenho mecânico quando comparados às misturas de concreto de controle, considerando à resistência à compressão e absorção de água capilar.

Alves, Vieira, De Brito \& Correia, (2014) avaliaram o efeito da incorporação de cerâmicas recicladas como agregados no concreto, sendo elas oriundas de tijolos e louças sanitárias trituradas. Os parâmetros mecânicos avaliados foram resistência à compressão, resistência à tração, módulo de elasticidade e resistência à abrasão. A substituição do agregado natural pelo reciclado corresponderam a $20 \%, 50 \%$ e $100 \%$. Os resultados obtidos mostraram que o concreto que contemplou os tijolos triturados como agregado apresentou melhor desempenho estrutural quando comparado ao concreto feito com louças sanitárias recicladas, isso em comparação com o concreto de referência.

Brune (2017), avaliou os efeitos da incorporação do RCV, através do desempenho mecânico do concreto com substituição total e parcial do cimento (15\% e 25\%) pelo resíduo 
supracitado. Os resultados mostraram que houve uma diminuição da resistência à compressão simples nas substituições de $15 \%$ e $25 \%$, portanto, possuindo $88 \%$ e $74 \%$, respectivamente, da resistência da amostra de controle. Em relação à resistência a tração por compressão diametral, as amostras detiveram $80 \%$ e $68 \%$, respectivamente, da resistência da amostra de referência.

Santos, Castro \& Gonçalves (2018), avaliaram a utilização do RCV na produção de concreto autoadensável. Considerando, portanto, as propriedades de autoadensabilidade no estado fresco, ou seja, no concreto com material recém-misturado, bem como as propriedades físicas e mecânicas no estado endurecido. Logo, os resultados inferiram que o material possui um grande potencial tanto na produção de cimento, quanto para utilização na produção de concreto autoadensável, melhorando a estabilidade, bem como as propriedades físicas e mecânicas dos concretos.

Diante do exposto, este estudo visa analisar a influência da substituição do agregado miúdo (areia natural) pelo RCV, através da Resistência à Compressão Simples (RCS) e do consumo de cimento Portland no concreto, de modo a reduzir os custos, bem como os impactos associados ao uso do concreto, tendo em vista o seu largo consumo.

\section{Metodologia}

\subsection{Materiais}

As pesquisas são necessárias para se trazer novos saberes para a sociedade como preconiza Pereira et al. (2018). No presente estudo, os materiais utilizados na pesquisa foram o cimento Portland, mais especificamente o CPV ARI, em decorrência da sua alta resistência inicial. A água, proveniente do sistema de abastecimento do Instituto Federal do Ceará Campus Juazeiro do Norte, bem como os agregados, sendo estes os resíduos de cerâmica vermelha, a areia natural e a brita.

O resíduo cerâmico utilizado nesse estudo é oriundo de uma indústria cerâmica localizada no município de Crato/CE. Os resíduos coletados estavam no formato de tijolos cerâmicos (paralelepípedos), sendo estes fraturados ou ainda danificados. Os RCV foram submetidos à trituração através de um compactador mecânico, de modo a obter um material passante na peneira $4,75 \mathrm{~mm}$ e retido na peneira $0,075 \mathrm{~mm}$, intervalo este, que caracteriza o material como agregado miúdo. O agregado miúdo natural utilizado foi uma areia de rio quartzosa, e como agregado graúdo utilizou-se a brita 1, de origem basáltica. Esses por sua 
vez, foram caracterizados, conforme o descrito nas NBR 7211/2009 (Agregados para concreto Especificação), NBR 52/2009 (Agregado miúdo - Determinação da massa específica e massa específica aparente) e NBR 248/2003 (Agregados- Determinação da composição granulométrica).

Todos os agregados (graúdo, miúdo natural e miúdo reciclado) foram secos em estufa, sob temperatura de $105 \pm 5^{\circ} \mathrm{C}$ por $24 \mathrm{~h}$, e submetidos a ensaios de determinação da composição granulométrica e massa específica e Fluorescência de Raios X (FRX), sendo este último realizado somente com o agregado reciclado.

\subsection{Método}

Em se tratando da produção dos concretos, seguiu-se o método de dosagem do Instituto de Pesquisas Tecnológicas (IPT/EPUSP), descrito por Helene \& Terzian (1992). Para tanto, foram escolhidos três tipos de traços 1:3,5; 1:5,0 e 1:6,5 e para cada traço, foram feitas três dosagens com substituição do agregado miúdo natural nas porcentagens de $0 \%$, $50 \%$ e $100 \%$, totalizando nove misturas de concreto. Na Tabela 1 apresentam-se as substituições analisadas na pesquisa.

Tabela 1: Substituições analisadas.

\begin{tabular}{c|c|c|c}
\hline Sigla & Traços & $\begin{array}{c}\text { \%Agregado } \\
\text { miúdo } \\
\text { Natural }\end{array}$ & $\begin{array}{c}\text { \%Agregado } \\
\text { miúdo } \\
\text { Reciclado }\end{array}$ \\
\hline CREF & $1: 3,5$ & & \\
& $1: 5,0$ & 100 & 0 \\
& $1: 6,5$ & & \\
CS50 & $1: 3,5$ & & 50 \\
& $1: 5,0$ & 50 & \\
& $1: 6,5$ & & \\
CS100 & $1: 3,5$ & & 100 \\
& $1: 5,0$ & 0 & \\
\hline
\end{tabular}

Fonte: Autores.

Os concretos contendo o resíduo da indústria cerâmica, com substituição parcial (CS50) e total (CS100) foram avaliados em relação aos concretos feitos sem a substituição do agregado natural, sendo este o concreto de referência (CREF). Os concretos foram fabricados com teor de argamassa de 53\% e sua trabalhabilidade foi analisada através do abatimento de 
tronco de cone (slump test) de $80 \pm 10$. Na Tabela 2 são apresentados os traços, slump e a relação água/cimento $(\mathrm{a} / \mathrm{c})$ dos concretos fabricados.

Tabela 2: Concretos fabricados

\begin{tabular}{|c|c|c|c|}
\hline \multicolumn{2}{|c|}{ Traço } & Slump & $a / c$ \\
\hline \multirow{3}{*}{ CREF } & $1: 3,5$ & $70 \mathrm{~mm}$ & 0,47 \\
\hline & $1: 5,0$ & $75 \mathrm{~mm}$ & 0,64 \\
\hline & $1: 6,5$ & $75 \mathrm{~mm}$ & 0,81 \\
\hline \multirow{3}{*}{ CS50 } & $1: 3,5$ & $88 \mathrm{~mm}$ & 0,54 \\
\hline & $1: 5,0$ & $79 \mathrm{~mm}$ & 0,76 \\
\hline & $1: 6,5$ & $75 \mathrm{~mm}$ & 0,80 \\
\hline \multirow{3}{*}{ CS100 } & $1: 3,5$ & $75 \mathrm{~mm}$ & 0,63 \\
\hline & $1: 5,0$ & $72 \mathrm{~mm}$ & 0,84 \\
\hline & $1: 6,5$ & $79 \mathrm{~mm}$ & 1,05 \\
\hline
\end{tabular}

Fonte: Autores.

Convém ressaltar que mesmo em decorrência da distinção entre as massas específicas dos agregados (miúdo natural e reciclado), não houve ajuste na proporção de agregado reciclado para que o volume de ambos fosse o mesmo. Dessa forma, a substituição foi feita somente com base na quantidade igualitária de massa e não de volume dos agregados.

Foram moldados dois corpos de prova cilíndricos para cada dosagem, seguindo o estabelecido na NBR 5738/2015 (Concreto - Procedimento para moldagem e cura de corpos de prova), totalizando dezoito corpos de prova com dimensões cilíndricas de $100 \mathrm{~mm}$ de diâmetro e $200 \mathrm{~mm}$ de altura. Após $24 \mathrm{~h}$ da moldagem os corpos de prova foram desmoldados e submetidos à cura, totalmente imersos em água por 28 dias, conforme demonstrado na Figura 1.

Figura 1: Corpos de provas imersos em água.

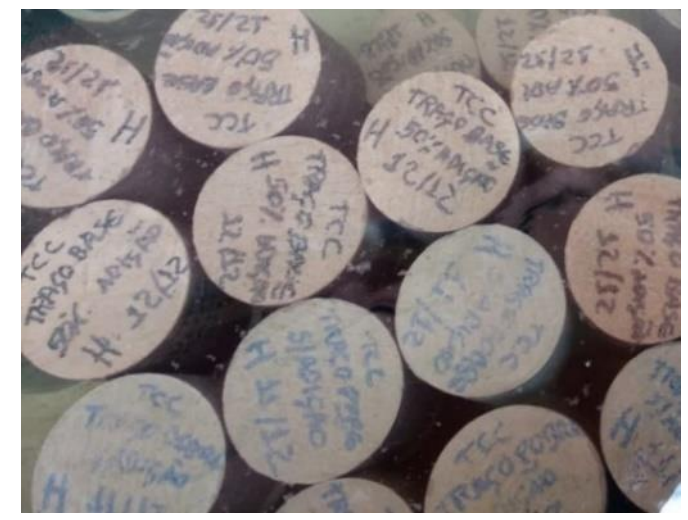

Fonte: Autores. 
Após o período de cura supracitado, os corpos de prova foram submetidos ao ensaio de Resistência à Compressão Simples (RCS), detalhado na Figura 2, seguindo as recomendações da NBR 5739/2018 (Concreto - Ensaio de compressão de corpos de prova cilíndricos).

Figura 2: Corpos de prova submetidos ao ensaio de RSC .
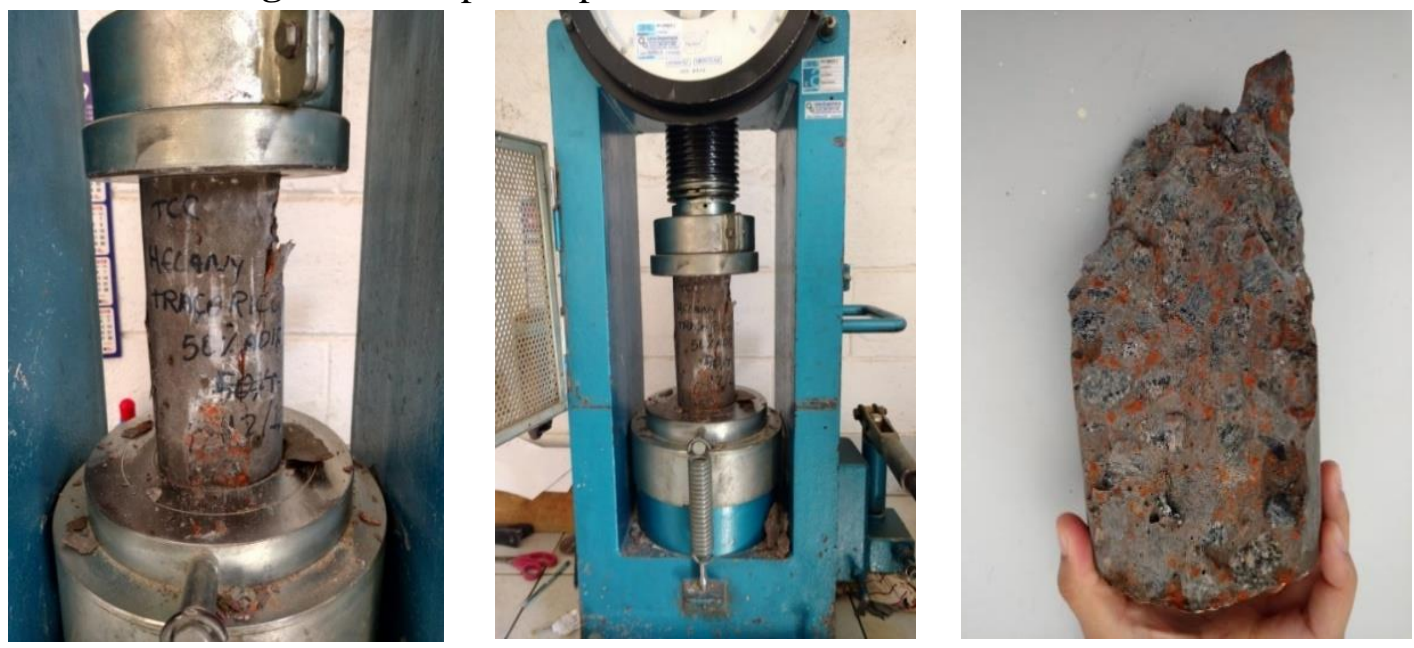

Fonte: Autores.

A análise foi realizada no laboratório de materiais da empresa Polimix Concreto. O capeamento dos corpos de prova foi realizado em uma retífica, também na empresa citada.

O consumo de cimento Portland, foi determinado através da Equação 1, de acordo com o consumo de cimento de Molinari, ressalta-se que a dosagem foi realizada sempre considerando a massa dos materiais.

$$
\mathrm{C}=\frac{\mathrm{ME}}{1+\mathrm{a}+\mathrm{p}+(\mathrm{a} / \mathrm{c})}
$$

Onde, $\mathrm{C}=$ consumo de cimento em massa; $\mathrm{ME}=$ massa específica do concreto; $\mathrm{a}=$ areia, $\mathrm{p}$ : brita; a/c: relação água/ cimento.

\section{Resultados e Discussão}

Na região do Cariri cearense há uma carência de materiais para serem usados como agregados finos em concretos, assim neste trabalho investigou-se a melhor forma de utilizar determinados percentuais de RCV como substituição á areias, comumente utilizadas na fabricação de concretos. Busca-se assim, entender qual a melhor configuração de dosagem 
Research, Society and Development, v. 9, n. 7, e357974145, 2020

(CC BY 4.0) | ISSN 2525-3409 | DOI: http://dx.doi.org/10.33448/rsd-v9i7.4145

com a substituição de areia por RCV, através do comportamento mecânico e consumo de cimento. Na Figura 3 e na Tabela 3 são apresentados os resultados inerentes a granulometria dos agregados.

Figura 3: Curva granulométrica dos agregados.

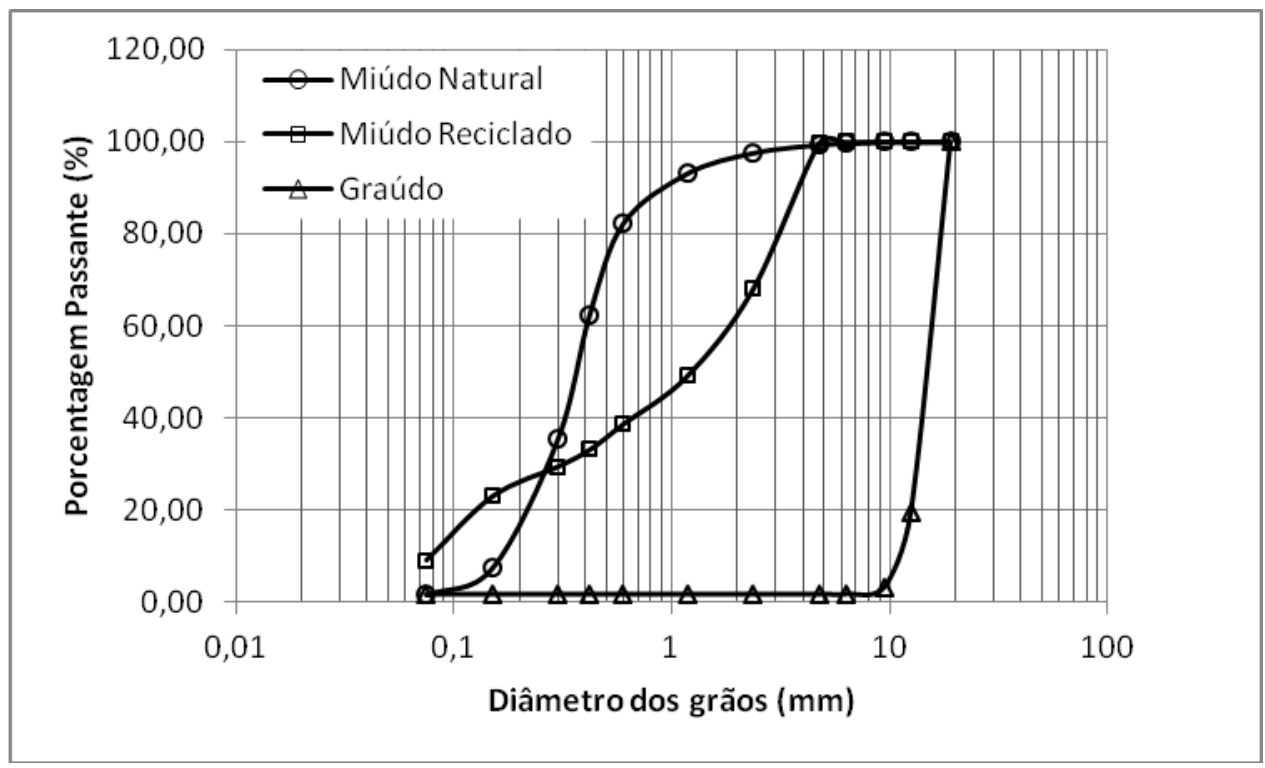

Fonte: Autores.

Tabela 3: Características físicas dos agregados.

\begin{tabular}{lccc}
\hline & Miúdo Natural & Miúdo Reciclado & Graúdo \\
\hline Módulo de Finura & 1,85 & 2,92 & 6,98 \\
Dimensão Máxima & 2,36 & 4,75 & 10 \\
Característica $(\mathrm{mm})$ & & & \\
Massa Específica $\left(\mathrm{g} / \mathrm{cm}^{3}\right)$ & 2,66 & 2,42 & 2,71 \\
\hline
\end{tabular}

Fonte: Autores.

Com base no módulo de finura dos agregados miúdos (areia e resíduo cerâmico), percebeu-se que os dois se enquadram nos limites da NBR 7211/2009. A areia encontra-se na zona inferior utilizável (módulo de finura ente 1,55 e 2,2), e o resíduo cerâmico na zona superior utilizável (módulo de finura entre 2,9 e 3,50).

De acordo com as curva granulométricas dos agregados percebeu-se que o resíduo não se enquadra perfeitamente dentro dos limites da referida norma, mas a maioria dos prontos da 
Research, Society and Development, v. 9, n. 7, e357974145, 2020

(CC BY 4.0) | ISSN 2525-3409 | DOI: http://dx.doi.org/10.33448/rsd-v9i7.4145

curva se encaixa nos limites e o miúdo (areia) se enquadra perfeitamente nos limites da NBR 7211/2009, conforme o apresentado na Figura 4.

Figura 4: Curva granulométrica do agregado miúdo e limites da NBR 7211.

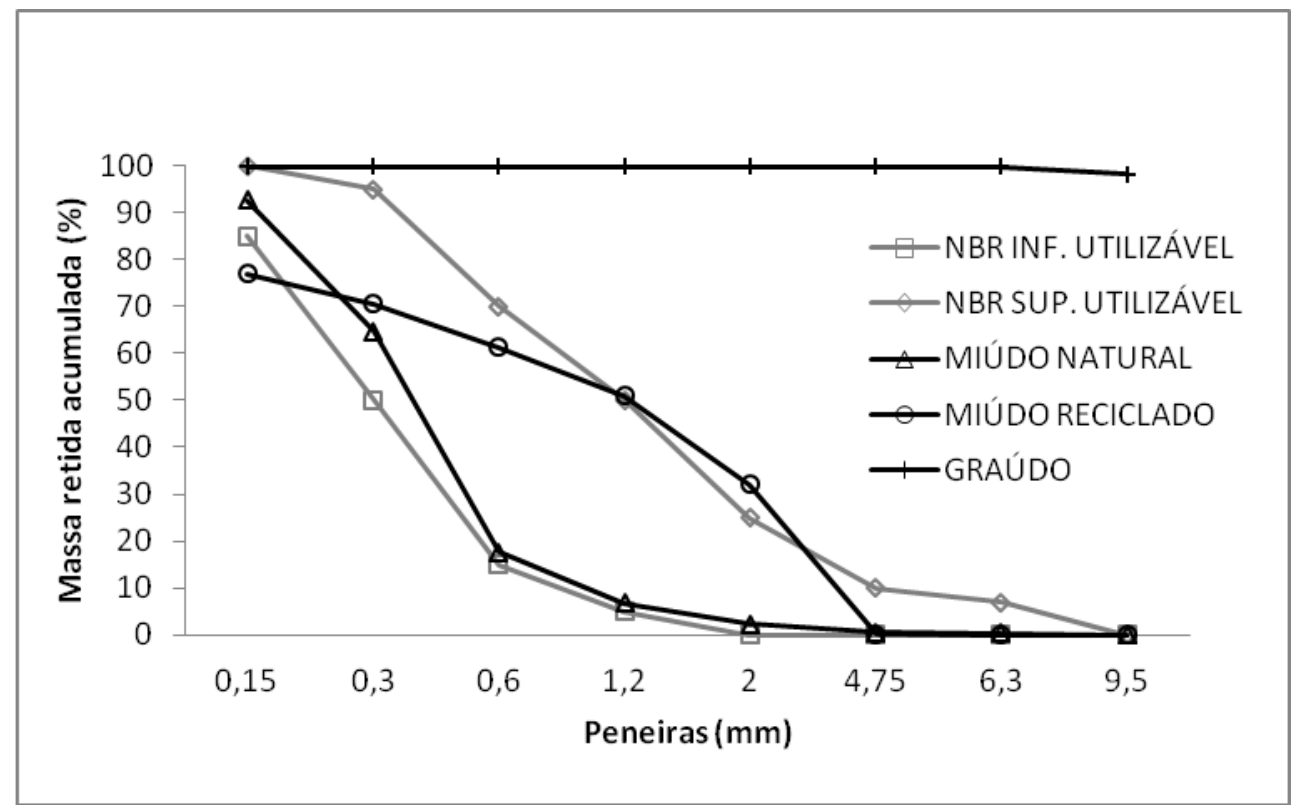

Fonte: Autores.

A composição mineralógica do resíduo de cerâmica vermelha foi determinada através do ensaio de Fluorescência de Raios X (FRX) em três amostras do material. Com a média dos resultados elaborou-se a Tabela 4 que mostra as porcentagens em óxidos contidos no resíduo.

Tabela 4: Composição do resíduo cerâmico (FRX).

\begin{tabular}{c|c}
\hline Fórmula & Concentração (\%) \\
\hline $\mathrm{SiO}_{2}$ & 40,32 \\
$\mathrm{Al}_{2} \mathrm{O}_{3}$ & 9,17 \\
$\mathrm{Fe}_{2} \mathrm{O}_{3}$ & 11,18 \\
$\mathrm{~K}_{2} \mathrm{O}$ & 4,52 \\
$\mathrm{TiO}_{2}$ & 1,36 \\
$\mathrm{CaO}$ & 1,18 \\
$\mathrm{BaO}$ & 0,11 \\
$\mathrm{Cl}$ & 0,17 \\
$\mathrm{P}_{2} \mathrm{O}_{5}$ & 0,42 \\
\hline
\end{tabular}

Fonte: Autores. 
A composição química dos resíduos cerâmicos triturados infere que o agregado reciclado possui um teor de $\mathrm{SiO}_{2}, \mathrm{Al}_{2} \mathrm{O}_{3}$ e $\mathrm{Fe}_{2} \mathrm{O}_{3}$ de $68,43 \%$, atendendo o exposto na NBR 12653/2014, para o uso como material pozolânico. Portanto, se enquadrando na classe E de pozolanicidade.

Em relação à RCS e ao consumo de cimento, priorizou-se um abatimento de tronco de cone de $80 \pm 10$ e um teor de argamassa de $53 \%$ para todos os concretos preparados, não sendo fixada a relação água/cimento e a resistência característica do concreto. Na Figura 5 apresentam-se os resultados do referido ensaio em cada concreto.

Figura 5: Resistência à compressão simples dos concretos.

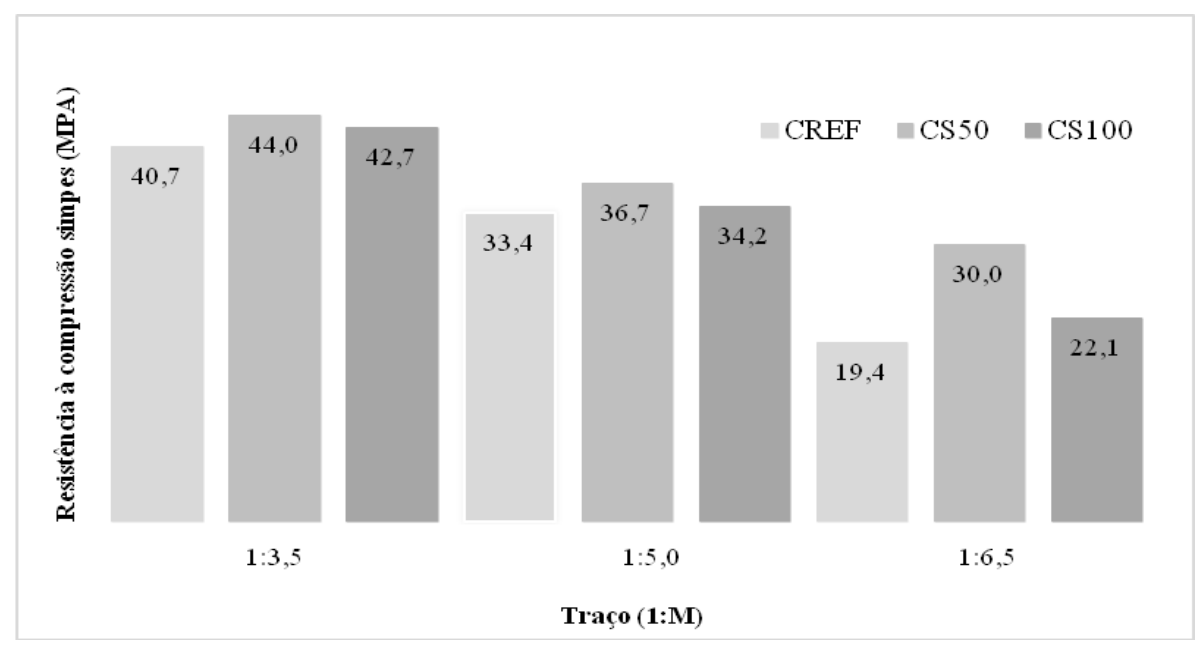

Fonte: Autores.

A partir do registro das massas específicas dos concretos (ME), no momento das dosagens, foi possível a execução do cálculo de consumo de cimento dos concretos.

Na Figura 6 apresenta-se o consumo de cimento em relação ao traço utilizado em todas as misturas preparadas. 
Research, Society and Development, v. 9, n. 7, e357974145, 2020

(CC BY 4.0) | ISSN 2525-3409 | DOI: http://dx.doi.org/10.33448/rsd-v9i7.4145

Figura 6: Consumo do cimento conforme o traço.

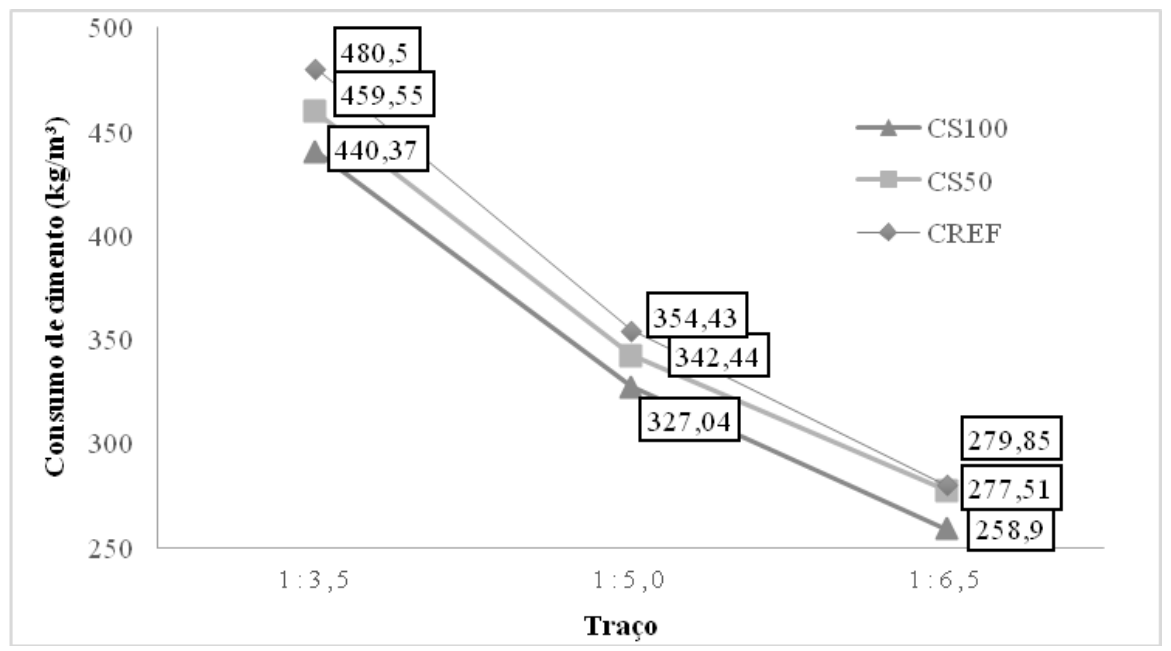

Fonte: Autores.

Na Figura 7, mostram-se, para os três traços realizados, a variação da RCS dos concretos em função do consumo de cimento Portland.

Figura 7: Consumo do cimento conforme a resistência à compressão.

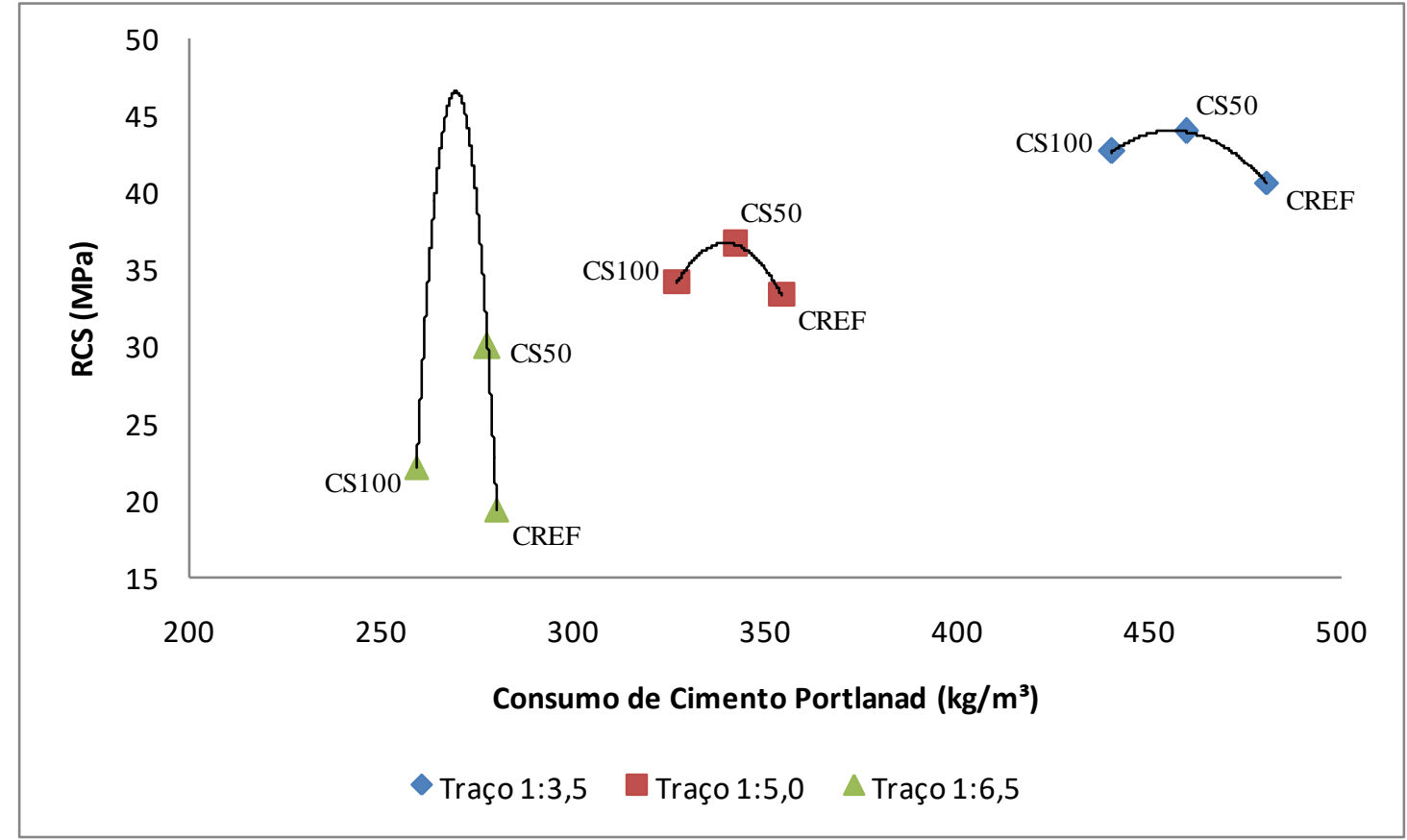

Fonte: Autores.

De acordo com as Figuras 6 e 7, é possível observar que os concretos CS50 e CS100 tiveram melhor desempenho do que o CREF, em relação à RCS e ao consumo de cimento. Os 
concretos produzidos com substituição parcial tiveram melhor performance no consumo de cimento e na RCS do que o de permutação total.

A Figura 7 mostra que, para os percentuais de 50\% e 100\% de substituição de agregado miúdo pelo RCV, o consumo de cimento Portland diminui e a RCS aumenta em relação ao traço de referência. Contudo, identifica-se que a RCS dos concretos CS50 são as mais elevadas. Assim, pode-se caracterizar que o a substituição de 50\% do agregado miúdo por RCV é o valor ótimo no concreto, pois as RCS são máximas para as dosagens dos três traços.

A partir da Figura 7 também é possível observar que quanto mais ricos em cimento Portland são os traços, a variação da RCS em relação ao consumo de cimento Portland diminui. Ainda observa-se que os valores de RCS aumentaram em maior percentual nos traços mais pobres, quando comparados aos traços de referência na substituição de RCV (50\%), mostrando assim que para um menor consumo de cimento é possível alcançar maiores variações dos valores de RCS substituindo o agregado miúdo pelo RCV.

\section{Considerações Finais}

Pode-se inferir que a substituição do agregado natural pelo agregado reciclado apresentou influência, de forma positiva, na resistência à compressão e no consumo de cimento. A utilização do RCV teve influência considerável na demanda de água dos concretos produzidos, o concreto CS100 foi o que apresentou melhor desempenho em relação ao menor consumo de cimento e o CS50 apresentou maior RCS. Verificou-se também que a substituição total do agregado natural pela cerâmica vermelha modificou a coloração dos concretos no estado fresco, atribuindo-lhes uma cor avermelhada, mas que no estado endurecido essa cor não fica tão evidente, assemelhando-se a cor acinzentada dos concretos CREF.

Assim, os melhores resultados encontrados de RCS foram dos concretos CS50 para os traços $(1: 6,5),(1: 5,0)$ e (1:3,5) com 30,0 MPa, 36,7 MPa e 44,0 MPa, respectivamente. Já em relação ao consumo de cimento, os concretos CS100 tiveram os melhores desempenhos, sendo que, para os traços $(1: 6,5),(1: 5,0)$ e $(1: 3,5)$ foram consumidos, respectivamente 258,9 $\mathrm{kg} / \mathrm{m}^{3}, 327,04 \mathrm{~kg} / \mathrm{m}^{3}$ e $440,37 \mathrm{~kg} / \mathrm{m}^{3}$.

Pode-se concluir que o resíduo de cerâmica vermelha analisado, se mostra como um material alternativo potencialmente viável de ser utilizado na fabricação de concretos, atribuindo-lhe aprimoramento em suas características de resistência à compressão e consumo 
de cimento. $\mathrm{O}$ que possibilitou essas evidentes influências causadas pelo resíduo cerâmico, em relação à resistência à compressão, foram suas características física, pois seus grãos possuem superfície áspera, a qual propicia uma melhor ligação entre a matriz de cimento e os agregados reciclados e, química, pois apresenta características que o classifica como um material pozolânico.

A massa específica do agregado reciclado proporcionou uma diminuição do consumo de cimento, justificando-se pelo fato de que quanto maior a massa específica dos agregados maior o consumo de cimento, logo os concretos produzidos com RCV possuem menor consumo de cimento, pois a massa específica do agregado reciclado é inferior ao do agregado natural.

Finalmente, para analisar com mais acurácia o efeito da adição do RCV em concretos, com vista a verificar a sua pozolanicidade, seria necessário a realização de imagens via microscopia eletrônica de varredura (MEV) com espectroscopia de energia dispersiva (EDS).

\section{Referências}

Alves, AV, Vieira, TF, Brito, J \& Correia, JR. (2014). Mechanical properties of structural concrete with fine recycled ceramic aggregates. Construction and Building Materials, 64 (1), 103-113. doi:10.1016 / j.conb uildmat.2014.04.037.

ABNT. (2003). NBR NM 248: Agregados - Determinação da composição granulométrica, Rio de Janeiro, 2003. Associação Brasileira de Normas Técnicas.

ABNT. (2009a). NBR NM 52: Agregado miúdo - Determinação da massa específica e massa específica aparente, Rio de Janeiro, 2009. Associação Brasileira de Normas Técnicas.

ABNT. (2009b). Associação Brasileira de Normas Técnicas. NBR 7211: Agregados para concreto - Especificação, Rio de Janeiro, 2009. Associação Brasileira de Normas Técnicas.

ABNT. (2014). NBR 12653 - Materiais pozolânicos, Rio de Janeiro, 2014. Associação Brasileira de Normas Técnicas.

ABNT. (2015). NBR 5738: Concreto - Moldagem e cura de corpos-de-prova cilíndricos ou prismáticos de Concreto, Rio de Janeiro, 2015. Associação Brasileira de Normas Técnicas. 
ABNT. (2018). NBR 5739 - Concreto - Ensaio de compressão de corpos-de-prova cilíndricos, Rio de Janeiro, 2018. Associação Brasileira de Normas Técnicas.

Banco do Nordeste do Brasil. (2010). Informe setorial de cerâmica vermelha. Recuperado de https://www.bnb.gov.br/documents/88765/89729/ano4_n21_informe_setorial_ceramica_verm elha.pdf/66eb35dc-dd49-420d-a921-26e9efc320d9.

Betat, EF, Pereira, FM. \& Verney, JCK. (2009). Concretos produzidos com resíduos do beneficiamento de ágata: avaliação da resistência à compressão e do consumo de cimento. Matéria, 14 (3), 1047-1060. doi:10.1590/S1517-70762009000300016.

Brune, C. (2017). Utilização de resíduo de cerâmica vermelha como substituição parcial do cimento em concretos. Trabalho de Conclusão de Curso. Universidade Regional do Noroeste do Estado do Rio Grande do Sul.

Cabalar, AF, Hassan, DI \& Abdulnafaa, MD.(2016). Use of waste ceramic tiles for road pavement subgrade. Road Materials and Pavement Design, 18 (4), 882-896. doi: 10.1080/14680629.2016.1194884.

Cabral, AEB, Schalch, V, Dal Molin, DCC \& Ribeiro, JLD. (2009). Desempenho de concretos com agregados reciclados de cerâmica vermelha. Cerâmica, 55 (336), 448-460. doi:10.1590/S0366-69132009000400016.

Couto, AF, Cezario, NS, Morales, G \& Toralles, BM. (2018). Efeitos da substituição parcial do agregado miúdo por cinza de madeira de eucalipto nas propriedades das argamassas. Revista de Engenharia Civil IMED, 5 (2), 84-98. doi:10.18256/23586508.2018.v5i2.2571.

Costa, JS \& Favini, AC. (2008). Resíduo de cerâmica vermelha como agregado alternativo para construção civil. In: $2^{\circ}$ jornada da produção científica da educação profissional $e$ tecnológica da região centro oeste. Cuiab 
García. E, Junior, MC, Quarcioni, VA \& Chotoli, FF. (2015). Avaliação da atividade pozolânica dos resíduos de cerâmica vermelha. Cerâmica, 61 (358), p. 251-258. doi: 10.1590/0366-69132015613581847.

García, JL. (2014). Empleo de residuos de concreto en la construccion de carpetas asfalticas. Master Thesis. Universidad Nacional Autonoma de Mexico.

Gonçalves, JP. 2007. Utilização do resíduo da indústria cerâmica para produção de concretos. Revista Escola de Minas, 60 (4). 639-644. doi:10.1590/S0370-44672007000400009.

Helene, P \& Terzian, P. (1992). Manual de dosagem e controle do concreto, Brasília: Pini. Lima, SA, Sales, A, Almeida, FCR, Moretti, JP \& Portela, KF. (2012). Concretos com cinza do bagaço da cana-de-açúcar: avaliação da durabilidade por meio de ensaios de carbonatação e abrasão. Ambiente Construído, 11 (2), 201-212. doi:10.1590/S1678-86212011000200014.

Leite, MB. (2001). Avaliação de propriedades mecânicas de concretos produzidos com agregados reciclados de resíduos de construção e demolição. Tese de Doutorado. Universidade Federal do Rio Grande do Sul.

Macedo, RS, Menezes, RR, Neves, GA \& Ferreira, HC. (2008). Estudo de argilas usadas em cerâmica vermelha, Cerâmica 54 (332), 411-417. doi:10.1590/S0366-69132008000400005.

Melo, KA. (2005). Contribuição à dosagem de concreto auto-adensável com adição de filer calcário. Dissertação de Mestrado. Universidade Federal de Santa Catarina.

Oliveira, LAP, Gomes, JPC \& Santos, PMS. (2012). The potential pozzolanic activity of glass and redclay ceramic waste as cement mortars components. Construction and Building Materials, 31 (1),197- 203. doi:10.1016/j.conbuildmat.2011.12.110.

Pacheco-Torgal, F \& Jalali, S. (2010). Reusing ceramic wastes in concrete. Construction and Building Materials, 24 (5), 832-838. doi: 10.1016/j.conbuildmat.2009.10.023.

Palhares, RA, Pereira, ARS, Cabral, KC \& Nobrega, AKC. (2016). Avaliação da atividade pozolânica dos resíduos de cerâmica vermelha produzidos no Vale do Assú/RN. In: $22^{\circ}$ 
Congresso Brasileiro de Engenharia e Ciências dos Materiais. 2016, (pp.1045-1056). Natal, Rio Grande do Norte.

Passos, L \& Moreno Jr, AL. (2018). Estudo da tensão de aderência entre barras de aço e concretos com agregados de resíduos de cerâmica vermelha. Ambiente Construído, 18 (4), 361-375. doi:10.1590/s1678-86212018000400310.

Pereira, AM, Silva, CAR, Queiroz, DC, Moraes, MJB, Melges, JLP, Tashima, MM \& Akasaki, JL. (2015). Estudo das propriedades mecânicas do concreto com adição de cinza de casca de arroz. Revista Matéria, 20 (1), 227-238. doi:10.1590/S1517-707620150001.0023.

Pereira, AS, Shitsuka, DM, Parreira, FJ \& Shitsuka, R. (2018). Metodologia da pesquisa científica. [e-book]. Santa Maria. Ed. UAB/NTE/UFSM. Acesso em: 15 maio 2020.

Disponível em:

https://repositorio.ufsm.br/bitstream/handle/1/15824/Lic_Computacao_MetodologiaPesquisa-Cientifica.pdf?sequence $=1$.

Possa, SA \& Antunes, EGP. (2016). Proposta de reutilização do resíduo de cerâmica vermelha proveniente da construção civil e demolições no município de Criciúma, SC. Revista Tecnologia e Ambiente, 22, 147-161. doi:10.18616 / ta.v21i0.2968.

Rosa, FR. (2011). Identificação e cadastramento de áreas de descarte de resíduos de construção e demolição na região da AMREC, Santa Catarina. Trabalho de Conclusão de Curso. Universidade Estadual de Santa Catarina.

Silva, PB. (2014). Estabilização de misturas de resíduos sólidos de demolição e da indústria cerâmica para uso em camadas de pavimentos viários. Tese de Doutorado. Universidade de São Paulo.

Silva, JPS \& Carvalho, SB. (2017). Uso de resíduos de cerâmica vermelha para o melhoramento de camadas de pavimentos de baixo tráfego em Palmas-TO. Revista Eletrônica de Gestão e Tecnologias Ambientais (GESTA), 5 (1), 41-52. doi: 109771/gesta.v5i1.17417. 
Santos, RFC, Castro, AL \& Goncalves, KM. (2018). Produção de concreto autoadensável incorporado com resíduo da indústria de cerâmica vermelha. Matéria (Rio J.)

23 (3), 1-15. doi: 10.1590/s1517-707620180003.0500.

Souza, WM, Lima, MMR, Xavier, IWP \& Ribeiro, AJA. (2018). Diagnóstico da geração de resíduos de cerâmica vermelha na Região Metropolitana do Cariri. In: congresso técnico científico de engenharia e da agronomia - CONTECC. Maceió-AL: CREA-AL.

Souza, WM, Xavier, IWP, Ribeiro, AJA \& Santos, MV. (2019). Resíduos de cerâmica vermelha como um material ambientalmente sustentável para uso na pavimentação. Revista Eletrônica de Gestão e Tecnologias Ambientais (GESTA), 7 (2), 202-213. Doi: 10.9771/gesta.v7i2.32540.

\section{Porcentagem de contribuição de cada autor no manuscrito}

Maria Helany Lima de Morais - $40 \%$

Wana Maria de Souza- 30\%

Antonio Júnior Alves Ribeiro - 30\% 